\title{
Magneto-optical studies of the correlation between interface microroughness parameters and the photoluminescence line shape in $\mathrm{GaAs} / \mathrm{Ga}_{0.7} \mathrm{Al}_{0.3} \mathrm{As}$ quantum wells
}

\author{
J. B. B. de Oliveira \\ Departamento de Física, Universidade Estadual Paulista, Av. Eng. Luiz. Edmundo C. Coube, s/n, Caixa Postal 473, 17033-360, Bauru, \\ SP, Brazil \\ E. A. Meneses \\ Instituto de Física Gleb Wataghin, Universidade Estadual de Campinas, Caixa Postal 6165, 13083-970 Campinas, SP, Brazil \\ E. C. F. da Silva \\ Instituto de Física da Universidade de São Paulo, Caixa Postal 66318, 05315-970, São Paulo, SP, Brazil
}

(Received 29 October 1998)

\begin{abstract}
In this work we analyze the relation between the interface microroughness and the full width at half maximum (FWHM) of the photoluminescence (PL) spectra for a $\mathrm{GaAs} / \mathrm{Ga}_{0.7} \mathrm{Al}_{0.3} \mathrm{As}$ multiple quantum well (QW) system. We show that, in spite of the complex correlation between the microscopic interface-defects parameters and the QW optical properties, the Singh and Bajaj model [Appl. Phys. Lett. 44, 805 (1984)] provides a good quantitative description of the excitonic PL-FWHM. [S0163-1829(99)01424-1]
\end{abstract}

The interfaces structural properties in quantum wells (QW's) have been extensively investigated, as they are extremely important for the high performance of the electronic and optoelectronic QW-based devices. ${ }^{1-3}$

Semiconductor heterostructures interfaces have been investigated by means of direct or indirect characterization techniques. Direct investigations of the interfacial quality have been obtained, for instance, by scanning tunneling microscopy, atomic force microscopy, and transmission electron microscopy (TEM). ${ }^{4}$ However, interfaces that are deep inside a semiconductor heterostructure are not accessible through direct investigation methods, so optical techniques (which indirectly probe the interfaces) have been intensively used in semiconductor-interface characterization in QW systems.

One of the most efficient sensors used in the optical characterization of interfacial quality, due to their sensitivity to the structural aspects of the interface on the atomic scale, is the behavior of confined excitons. ${ }^{5}$ In fact, an interesting subject of debate is the correlation between the excitonic optical properties and the microscopic configurations of the interfaces. ${ }^{6-8}$

The flat island model (FIM) associates the properties of the QW emission lines with the microscopic patterns of the interfaces. According to this model, when the excitons are confined in extensive and plane islands, which have larger dimensions than the exciton lateral dimension, the photoluminescence (PL) emission line splits. These splittings are due to different confinement potentials, which are associated with islands of different sizes. Moreover, each one of the split PL emission line is broadened due to confinementpotential fluctuations. These fluctuations are due to interface microroughness (defects with smaller extension than that of the lateral dimension of the confined exciton) and to alloy fluctuations, resulting in an inhomogeneous (Gaussian) broadening of the PL spectrum. In addition, the emission spectra are also broadened due to final-state interactions (Lorentzian broadening) and due to the thermal distribution of the carriers.

In the FIM, the QW has different widths that differ from each other by an integer multiple of monolayers (ML), resulting in several PL peaks with corresponding differences in energy. However, more recently, splittings in the PL spectra related to noninteger $\mathrm{ML}$ variations in the $\mathrm{QW}$ width were observed. ${ }^{9}$ The authors of Ref. 9 argued that for a realistic description of the interfaces it is necessary to use a Fourier transform of the two-dimensional interfaces, which provides a full specification of the roughness spectrum. This is the so-called bimodal model. The observation of microroughness on top of the macroscopically flat islands gives support to this model. ${ }^{10}$ Additionally, PL with spatial resolution ${ }^{11,12}$ and near-field spectroscopy ${ }^{13}$ showed that the PL line shape is composed by the superposition of many very narrow lines (some $\mu \mathrm{eV}$ wide) that are associated with excitons localization by different lateral-potential fluctuations. Then, the farfield spectrum, i.e., the traditionally observed PL spectrum, is interpreted as an envelope of the very narrow lines associated with defects of different sizes. ${ }^{4}$

In this work we add a contribution to this interesting discussion by showing that the FWHM of the emission spectra, obtained by standard PL spectroscopy, can be quantitatively correlated with the structural parameters of the microroughness localized at QW interfaces. Our conclusion is based on the comparison between the FWHM measured directly from our magneto-optical PL spectra and the theoretical FWHM obtained from the Singh and Bajaj model, which is based on the flat island picture and on thermodynamic arguments. ${ }^{1,14,15}$

The sample investigated in this work was one multiple quantum well structure grown by molecular beam epitaxy (MBE) on top of a GaAs (100) substrate. The structure starts with a $1.0-\mu \mathrm{m}$-thick undoped GaAs buffer layer followed by the active region, composed by $20 \mathrm{GaAs} / \mathrm{Ga}_{0.7} \mathrm{Al}_{0.3} \mathrm{As}$ quan- 


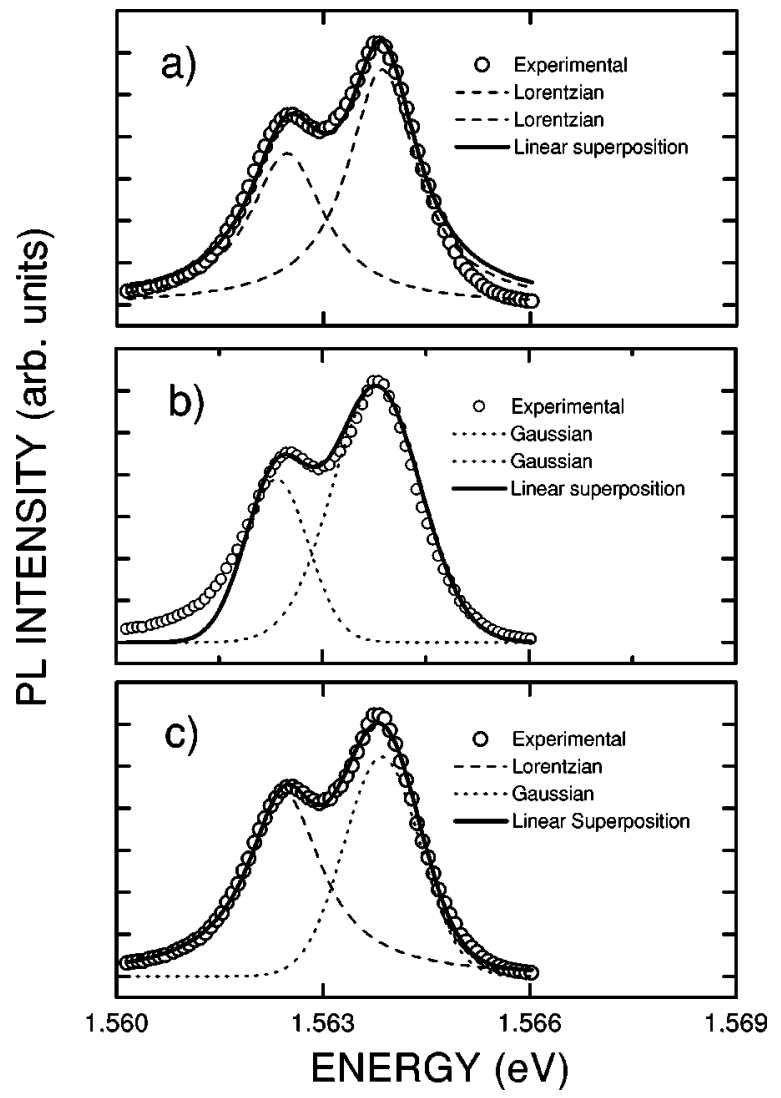

FIG. 1. Decomposition of the PL spectrum using: (a) two Lorentzian curves (dashed lines), (b) two Gaussian curves (dotted lines), (c) one Lorentzian and one Gaussian curve for the BE and the FE peaks, respectively. The open circles are the experimental data and the solid line represents the linear superposition of both fitted peaks.

tum wells. The wells and barriers are 80 and $300 \AA$ wide, respectively. The PL measurements were performed using an $\mathrm{Ar}^{+}$-ion laser as light source $\left(h \nu_{\mathrm{exc}}=2.41 \mathrm{eV}\right)$. The luminescence was dispersed by a $0.8-\mathrm{m}$ double monochromator and detected by a GaAs-cooled detector and a typical photocounter system. Magneto-optical measurements were carried out using the Faraday configuration within a $13 \mathrm{~T}$ superconducting magnet. Optical fibers coupled to a lens were used to transmit the laser beam to the sample and to collect the emitted light. The dimension of the laser spot on the sample was maintained constant with a diameter of approximately $200 \mu \mathrm{m}$.

Figures 1(a)-1(c) show a line-shape analysis of the PL spectrum of our sample measured at $2 \mathrm{~K}$ with low excitation density $\left(18 \mathrm{~mW} / \mathrm{cm}^{2}\right)$. PL and PLE spectra (not shown here) show a small Stokes shift of only $0.7 \mathrm{meV}$ indicating the good quality of the sample. In addition to the free exciton (FE) emission at $1.564 \mathrm{eV}$, another peak localized $1.5 \mathrm{meV}$ below the FE peak can also be observed in the spectrum of Fig. 1. Results of detailed PL and PLE measurements indicated that the low-energy peak was a photoinduced emission associated with excitons bound to defects localized at the QW interfaces, i.e., a bound exciton (BE). ${ }^{6,16,17}$

In order to analyze the line shape associated with the FE emission line, we decomposed the observed PL spectrum into its constituents using two Lorentzian curves [Fig. 1(a)], two Gaussian curves [Fig. 1(b)], and one Lorentzian and one Gaussian curve [Fig. 1(c)] to fit the BE and the FE emissions, respectively. It can be observed in Fig. 1(a) that the resulting curve (solid line) obtained by using the linear superposition of two Lorentzian curves does not fit the highenergy region of the experimental spectrum (open circles). On the other hand, the resulting curve obtained using two Gaussian curves [Fig. 1(b)] does not fit the low-energy region of the experimental spectrum. However, we can also observe in Fig. 1(c) that the linear superposition of a Lorentzian curve for the BE emission and a Gaussian curve for the $\mathrm{FE}$ emission provides a perfect adjust to the experimental data. This result indicates that the structural patterns of the interfaces dominate the FE emission, so the Lorentzian broadening can be neglected.

Using the Lifshitz treatment to study the excitation spectra of disordered alloys, Singh and Bajaj developed theoretical expressions correlating the dimensions of the microroughness localized at the QW interfaces and the alloy fluctuations with the FWHM of the excitonic emission line.

Following the description of Aksenov et al., ${ }^{7}$ when the broadening of the PL emission is determined by the quality of only one interface (in our case the interface formed by the growth of GaAs on top of the $\mathrm{Ga}_{0.7} \mathrm{Al}_{0.3} \mathrm{As}$ layer) and one considers an infinitely high barrier, the PL-FWHM associated with the microroughness at the interface $\left(\sigma_{\text {int }}\right)$ is described as

$$
\sigma_{\text {int }}=0.59 \frac{\delta_{1} \delta_{2}}{\mu L_{z}^{3} R_{\mathrm{ex}}^{2}},
$$

where $\delta_{1}$ and $\delta_{2}$ are, respectively, the height and the lateral dimension of the interface islands, $L_{z}$ is the QW width, $\mu$ is the reduced exciton mass in the direction perpendicular to the QW layer, and $R_{\text {ex }}$ is the exciton lateral dimension $\left(R_{\mathrm{ex}}\right.$ $=\sqrt{\left\langle\Psi\left|x^{2}+y^{2}\right| \Psi\right\rangle}$ ). Here it was considered that the averaged coverage of the interface assumed the most probable value of 0.5 . $^{7}$

Also, the Singh and Bajaj model describes the PL-FWHM associated with alloy fluctuation $\left(\sigma_{\text {alloy }}\right)$ as

$$
\sigma_{\text {alloy }}=2\left(\frac{1.4 x(1-x) r_{c}^{3}}{R_{\mathrm{ex}}^{3} P_{0}^{\mathrm{ex}}}\right)^{1 / 2}\left(\frac{\delta E_{\mathrm{ex}}}{\delta x}\right)_{x=x_{0}},
$$

where $r_{c}$ is the radius associated with the volume per cation (aproximately $2.5 \AA$ ), $E_{\mathrm{ex}}$ is the excitonic transition energy, $x$ is the $\mathrm{Al}$ concentration, $x_{0}$ is the nominal $\mathrm{Al}$ concentration (0.3 in our sample), and $P_{0}^{\mathrm{ex}}$ is the fraction of the exciton volume that penetrates in the barrier.

The total FWHM of the excitonic emission line $\sigma_{\text {tot }}$ is written as

$$
\sigma_{\text {tot }}=\sqrt{\sigma_{\text {int }}^{2}+\sigma_{\text {alloy }}^{2}},
$$

where both $\sigma_{\text {int }}$ and $\sigma_{\text {alloy }}$ depend on the exciton lateral dimension $R_{\mathrm{ex}}$. Since the value of $R_{\mathrm{ex}}$ can be modified by the application of an external magnetic field parallel to the growth direction, it is possible to probe the QW interfaces changing the intensity of the applied magnetic field $B$.

When an increasing magnetic field is applied along the growth direction of the QW sample, it leads to a decrease of 


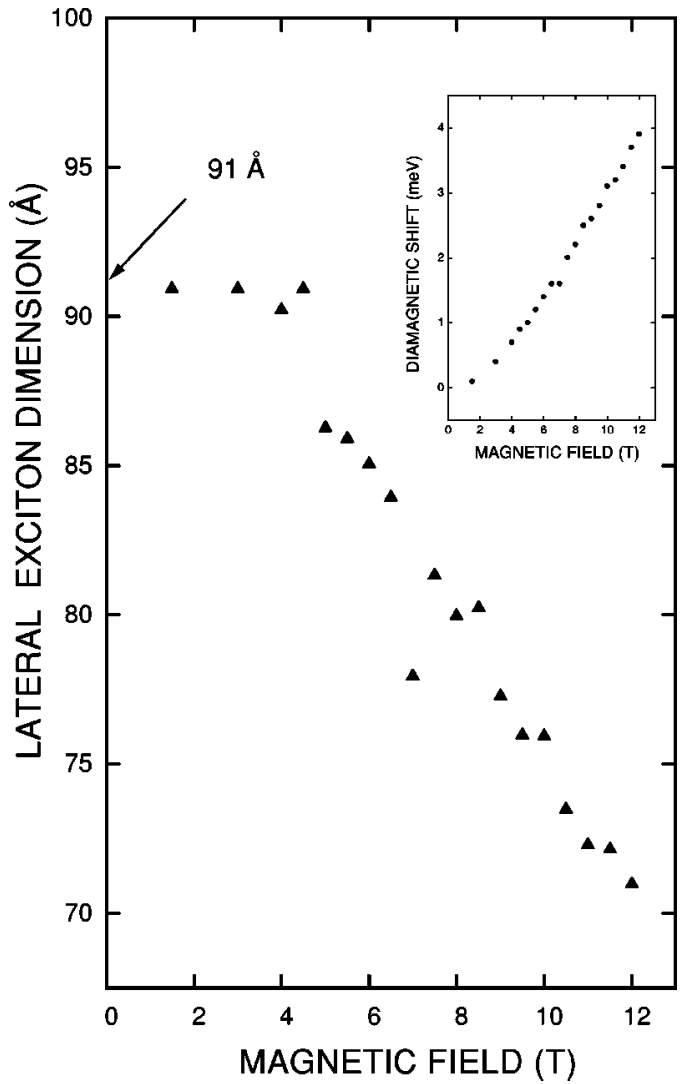

FIG. 2. Lateral exciton dimension $R_{\mathrm{ex}}$ as a function of the perpendicular magnetic field $B$. The inset shows the diamagnetic shift $[\Delta E(1 S)]$ as a function of $B$.

$R_{\text {ex }}$ due to an additional confinement induced by $B$. Moreover, the excitonic emission undergoes a diamagnetic shift $[\Delta E(1 S)]$, which can be written as $\Delta E(1 S)$ $=\left(e^{2} B^{2} / 8 \mu_{x y}\right) R_{\mathrm{ex}}^{2}$, for the fundamental excitonic state $(1 S) .{ }^{18}$ Here, $e$ is the elementary charge and $\mu_{x y}$ is the exciton in-plane mass $\left(\mu_{x y}=0.041 m_{0}\right.$ for our $\left.\mathrm{GaAs} / \mathrm{Ga}_{0.7} \mathrm{Al}_{0.3} \mathrm{As} \mathrm{QW}\right)$.

We performed magneto-PL measurements from 0 up to $12 \mathrm{~T}$ in the Faraday configuration as described earlier. The PL spectra obtained by applying different magnetic fields were decomposed using a Lorentzian and a Gaussian curve to simulate the $\mathrm{BE}$ and $\mathrm{FE}$ emission peak, respectively. The inset of Fig. 2 shows the diamagnetic shift as a function of $B$ obtained from our experimental data. From these results, keeping the zero magnetic field value of $\mu_{x y}$ and using the equation for $\Delta E(1 S)$ we calculated the exciton lateral dimension $\left(R_{\mathrm{ex}}\right)$ as a function of the magnetic field. The results are plotted in Fig. 2. As it was expected, $R_{\text {ex }}$ decreases for increasing magnetic field as a consequence of the additional magnetic confinement. In Fig. 2 we note that the extrapolation down to zero magnetic field indicates a value of approximately $91 \AA$ for $R_{\mathrm{ex}}$ at $B=0$. This value of the exciton dimension is in agreement with others obtained in the literature. ${ }^{19}$

Also, from the data of Fig. 2 we observe that $R_{\mathrm{ex}}$, and consequently the optical probe, assumes its maximun value at low magnetic field. Under that experimental condition, the excitonic PL-FWHM can be very sensitive to the alloy fluctuation due to the larger penetration of the excitonic wave

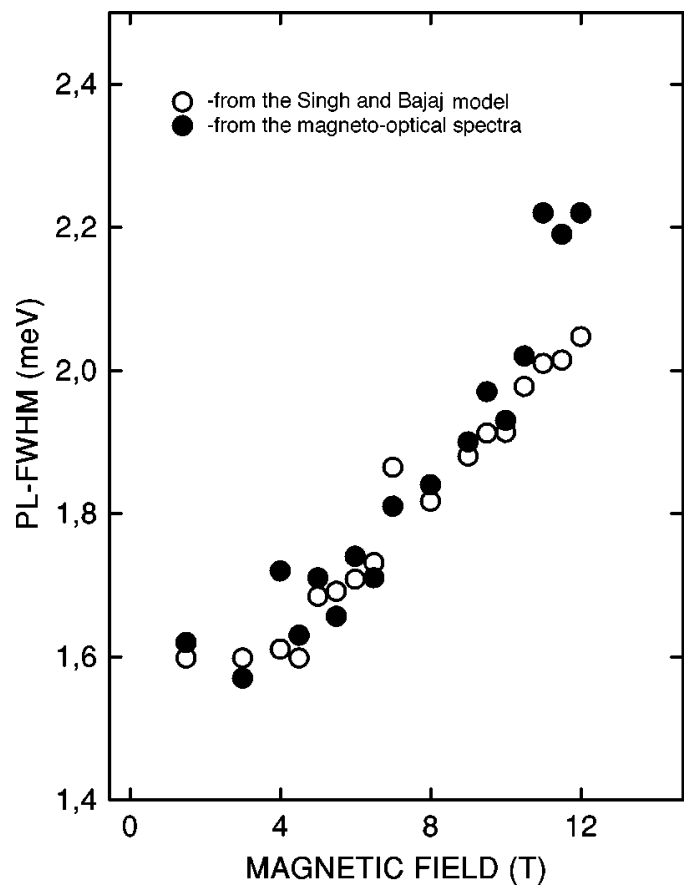

FIG. 3. PL-FWHM of the FE emission obtained from the decomposition of our magneto-optical data (solid circles) and values of $\sigma_{\text {int }}$ obtained from the Singh and Bajaj model (open circles).

function into the barrier. Therefore, before investigating the dependence of the PL-FWHM on $B$, we have estimated the maximum value that $\sigma_{\text {alloy }}$ assumes, i.e., at $B=0$.

In Eq. (2) we considered that the exciton fraction in the barrier at $B=0$ could be estimated as $P_{0}^{\mathrm{ex}}=1-L_{z}^{3} / R_{\mathrm{ex}}^{3}$, with $L_{z}=80 \AA$ and $R_{\mathrm{ex}}=91 \AA$, which gives $P^{\mathrm{ex}}=0.32$. On the other hand, we have calculated the dependence of $E_{\text {ex }}$ on $x$ and obtained $0.034 \mathrm{meV}$ for $\left(\delta E_{\mathrm{ex}} / \delta x\right)_{x=0.3} \cdot{ }^{20}$ With these values we found $\sigma_{\text {alloy }}=0.29 \mathrm{meV}$. Since we have measured $\sigma_{\text {tot }}=1.60 \mathrm{meV}$ [from the PL spectrum in Fig. 1(c)] we obtained $\sigma_{\text {int }}=1.57 \mathrm{meV}$ using Eq. (3). As a consequence, our calculations show that the alloy fluctuations have a minor influence on the broadening of the PL spectra of our sample, i.e., $\sigma_{\text {tot }} \cong \sigma_{\text {int }}$ as it was expected. Thus, for wider $\mathrm{QW}$ the amplitude of the excitonic wave function in the barrier will be negligible and the alloy-fluctuation effects can generally be discarded.

If we now assume in Eq. (1) that the island height is $\delta_{1}$ $=2.83 \AA$ (one GaAs ML), the reduced excitonic mass is $\mu=0.056 m_{0}$ (value determined from the Luttinger parameters for $\mathrm{GaAs}), \quad \sigma_{\mathrm{int}} \cong 1.6 \mathrm{meV}, L_{z}=80 \AA$, and $R_{\mathrm{ex}}$ $=91 \AA$ (for $B=0$ ), thus we obtain $\delta_{2}=33 \AA$ for the lateral dimension of the microroughness. This value is in good agreement with the one determined by TEM for $\mathrm{GaAs} / \mathrm{Ga}_{0.7} \mathrm{Al}_{0.3} \mathrm{As}$ QW. ${ }^{9}$

Using $\delta_{2}=33 \AA$ and the values of $R_{\text {ex }}$ plotted in Fig. 2, we can calculate $\sigma_{\text {int }}$ for each value of $B$ from the same Eq. (1), as shown in Fig. 3. In this figure we also plotted the values of the experimental FWHM that were obtained directly from the decomposition of our experimental magneto-PL spectra (solid circles). It can be seen that both experimental and theoretical data are in good agreement.

It is important to stress that the main aspect of the results of Fig. 3 is the ability of the model to correctly describe the 
increase of the PL-FWHM when the size of the optical probe is reduced with the increasing magnetic field.

In a standard PL measurement, where the light-spot diameter is large, i.e., without spatial resolution (as in our experiment), the measured PL spectrum represents a linear superposition of very narrow lines associated with the lateralpotential fluctuations. ${ }^{11}$ In this sense the lateral dimension $\delta_{2}$ extracted from the Singh and Bajaj equation has to be interpreted as an effective lateral-interface-microroughness size.

In conclusion, we showed in this work that in spite of the complexity of the structural characteristics of the QW interfaces, where defects with different length scales are present, the theoretical model of Singh and Bajaj provides a quantitative description of the broadening of the emission line due to the microroughness localized at the $\mathrm{Al}_{x} \mathrm{Ga}_{1-x} \mathrm{As} / \mathrm{GaAs}$ interface.

The authors acknowledge the Conselho Nacional de Desenvolvimento Científico e Tecnológico $(\mathrm{CNPq})$ and the Fundação de Amparo à Pesquisa de São Paulo (FAPESP) for financial support.
${ }^{1}$ J. Singh and K. K. Bajaj, J. Appl. Phys. 57, 5433 (1985).

${ }^{2}$ P. Borri, M. Gurioli, C. Colocci, F. Martelli, M. Capizzi, A. Patenè, and A. Polimeni, J. Appl. Phys. 80, 3011 (1996).

${ }^{3}$ K. Fujiwara, K. Kanamoto, and N. Tsukada, Phys. Rev. B 40, 9698 (1989).

${ }^{4}$ U. Jahn, S. H. Kwok, M. Ramsteiner, R. Hey, H. T. Grahn, and E. Runge, Phys. Rev. B 54, 2733 (1996).

${ }^{5}$ J. Christen and D. Bimberg, Phys. Rev. B 42, 7213 (1990).

${ }^{6}$ F. Martelli, A. Polimeni, A. Patanè, M. Capizzi, P. Borri, M. Gurioli, M. Colocci, A. Bosacchi, and S. Franchi, Phys. Rev. B 53, 7421 (1996).

${ }^{7}$ I. Aksenov, J. Kusano, Y. Aoyagi, T. Sugano, T. Yasuda, and Y. Segawa, Phys. Rev. B 51, 4278 (1995).

${ }^{8}$ R. F. Kopf, E. F. Schubert, T. D. Harris, and R. S. Becker, Appl. Phys. Lett. 58, 631 (1991).

${ }^{9}$ C. Warwick and R. F. Kopf, Appl. Phys. Lett. 60, 386 (1992).

${ }^{10}$ K. Fujiwara, R. Cingolani, and K. Ploog, J. Phys. IV 3, 307 (1993).

${ }^{11}$ K. Bruner, G. Abstreiter, G. Böhm, G. Träkle, and G. Weimann,
Appl. Phys. Lett. 64, 3320 (1994).

${ }^{12}$ J. B. Zhu, H. I. Jeon, E.-K. Suh, H. J. Lee, and Y. G. Hwang, Phys. Rev. B 52, 16353 (1995).

${ }^{13}$ H. F. Hess, E. Betzig, T. D. Harris, L. N. Pfeiffer, and K. W. West, Science 264, 1740 (1994).

${ }^{14}$ J. Singh, K. K. Bajaj, and S. Chaudhuri, Appl. Phys. Lett. 44, 805 (1984).

${ }^{15}$ A. Patanè, A. Polimani, M. Capizzi, and F. Martelli, Phys. Rev. B 52, 2784 (1995).

${ }^{16}$ V. Srinivas, Y. J. Chen, and E. C. C. Wood, Solid State Commun. 89, 611 (1994).

${ }^{17}$ J. B. B. Oliveira, E. A. Meneses, and E. C. F. Silva, Braz. J. Phys. 27/A, 193 (1997).

${ }^{18}$ W. Ossau, B. Jakel, E. Bangert, and G. Landwehr, Surf. Sci. 174, 188 (1986).

${ }^{19}$ R. Zimmerman and E. Runge, J. Lumin. 60-61, 320 (1994).

${ }^{20}$ G. Olegart, M. Proctor, D. Martin, F. Morier-Geraud, F.-K. Reinhart, B. Orschel, L. C. Andreani, and H. Rhan, Phys. Rev. B 49, 10456 (1994). 